

\section{Reviewer list 2016}

The editorial team of the Australasian Journal of Paramedicine would like to thank the following people for reviewing manuscripts published in 2016. Their generosity and contribution to the journal is greatly appreciated.

\begin{tabular}{|c|c|}
\hline Alison Porter & Joseph Acker \\
\hline Anat Audy & Joukje Oosterman \\
\hline Anders Bremer & Karen Pearson \\
\hline Ann Lazarsfeld-Jensen & Khadizah Haji Abdul-Mumin \\
\hline Anthony Kovac & Lisa McKenna \\
\hline Anuradha Joshi & Madison Brydges \\
\hline Bill Lord & Marc Colbeck \\
\hline Bryan Bledsoe & Nathan Puckeridge \\
\hline Cheryl Drewitz-Chesney & Nicholas Drey \\
\hline Dale Edwards & Olaf Drummer \\
\hline Dragan Ilic & Patricia Logan \\
\hline Elizabeth Thyer & Paul Davey \\
\hline Gwendolen Jull & Paul Jennings \\
\hline Habsah Hasan & Phillipa Malpas \\
\hline Jacob Sorensen & Scott Stewart \\
\hline James Thompson & Shane Knox \\
\hline Jenifer Pilgrim & Shaunagh Darroch \\
\hline Jera Kruja & Tania Johnston \\
\hline Jessica Paterson & Tom Quinn \\
\hline
\end{tabular}

